De ce tableau, il résulte que les différences décelées dans le lait normal et qui sont de 0,10 à $0,40 \%$, se montrent dans le lait desséché, presque dix fois plus grandes. Elles sont done provoquées par les constituants non identifiés, parmi lesquels l'acide citrique occupe la première place. Les grandes différences dans les analyses des laits pathologiques ne sont pas dues à une faute analytique, mais elles répondent à la présence dans le lait de substances dont la nature est encore inconnue ou difflcilement dosables.

\title{
LE TRANSPORT ACTUEL DU LAIT FRAIS
}

par le Professeur Dr B. LICHTENBERGER, de l'Institut de Laiterie de Kiel

Le chemin que, de nos jours, le lait doit, en général, parcourir avant d'arriver jusqu'au consommateur, est long. Le transport y joue un rôle important. Pour rendre l'exploitation du lait plus économique, il est nécessaire d'accorder toute notre attention à l'étude de ce transport et de ses diverses modalités.

Dans le problème du ravitaillement des grandes villes, il faut dans le transport du lait, distinguer trois temps:

$1^{\circ}$ De l'agriculteur au centre de ramassage ou dépôt central;

$2^{\circ}$ Du dépôt central à la laiterie de la Ville ;

$3^{\circ}$ De cette laiterie chez le consommateur.

Il n'y a pas encore longtemps que les centres de ramassage, comme stations de passage, manquaient. Le transport direct du producteur à la laiterie de la ville était seul à considérer, et il se faisait, ainsi, du reste que celui de la laiterie au consommateur, sans exception, avec des pots.

La situation a déjà changé dans certains pays, mais dans les autres, il ne saurait se passer beaucoup de temps avant que nous voyons s'y introduire les méthodes actuelles d'exploitation du lait. C'est ainsi que l'entrée en scène des dépôts centraux de ramassage, qui ne peuvent guère être évités, modifiera beaucoup l'aspect du transport.

Il n'est pas niable que la qualité du lait ne peut être bonne que si nous refroidissons celui-ci intensément aussitôt que possible, et dès la traite, autant que faire se peut.

Comme le refroidissement dans les centres de ramassage entraine nécessairement le mélange des laits, il est indispensable de fixer la qualité comme base du paiement du lait et nous ajouterons que les pots du producteur doivent être nettoyés aux centres de ramassage, parce que nous ne pouvons pas compter sur l'agriculteur pour nettoyer les pots comme il convient et, par suite, pour garantir la qualité du lait considéré aussi bien, évidemment, du côté hygiénique que du côté acidité.

Les centres de ramassage sont non seulement utiles pour assurer à la qualité du lait son uniformité, mais ils jouent un très grand rôle pour 
régler l'affluence du lait dans les Villes, pour éviter, en quelque sorte, l'inondation de celles-ci par un lait trop abondant, et enfin pour donner au prix de la matière première une stabilité plus grande. On atteindra ce but en faisant des dépôts centraux, non seulement des lieux de ramassage lesquels ont pour but de concentrer le lait en vue de l'évacuer sur la Ville, mais aussi des endroits où l'on opèrera la transformation du lait surabondant, soit en fromage, soit en beurre; on peut même y envisager la possibilité de l'établissement d'une condenserie ou d'une sècherie.

Dans ces conditions, la laiterie de Ville ne recevra que le lait qui lui est nécessaire et les excédents du centre de ramassage n'y parvenant point, bien des frais sont évités, et, ce qui est plus important peut-être encore, le lait n'aura pas à voyager, ce qui est toujours pour lui une occasion d'altération de sa qualité.

Le pot à lait restera probablement toujours le moyen de transport usuel dans le premier temps du transport de l'agriculteur au dépôt central et dans ce cas le dit transport est généralement effectué par une voiture à chevaux. Cependant aux Etats-Unis, on doit noter que l'automobile est devenu presque partout la voiture de ramassage. Il importe cependant de faire remarquer que dans des régions à productions disséminés où la voiture doit s'arrêter souvent pour recueillir ou pour rendre les pots, il y a là un mode de travail qui nuit beaucoup à l'emploi de l'automobile et à son économie.

Jusqu'à présent, dans tous les pays, le pot de fer étamé servait comme pot à lait, et changeaient seulemet, suivant les régions, la forme et la contenance du pot. Les Etats-Unis préfèrent par exemple le pot de 40 quarts (presque 50 litres) avec couvercle débordant sur le pourtour du col et faisant en quelque sorte l'effet d'un parapluie; l'Angleterre a des pots (churns) coniques pouvant contenir près de 80 à 100 litres. La Suède préfère le pot carré de 50 litres. En Allemangne, on se sert généralement d'un pot de 20 litres avec couvercle spécial, mais de plus en plus la tendance est d'employer des pots de 50 litres. En Autriche, on. s'en tient au pot courant avec un couvercle sous lequel on glisse une feuille de papier parcheminé, comme cela se fait d'ailleurs beaucoup aux Etats-Unis. Probablement, dans l'avenir, se mettra-t-on d'accord sur les pots et tendra-t-on à accepter un format unique, parce qu'on commence à comprendre un peu partout la grande valeur économique de formats acceptés une fois pour toutes d'une " norme ", d'un "standard " selon le langage américain.

Des changements seront également à prévoir dans la question du matériel. La durée des pots en fer étamé est relativement courte, si l'on tient à avoir un pot qui, jamais ne présente la moindre trace de rouille, celle-ci étant nuisible à la qualité du lait. Tous les efforts faits en vue d'éviter la rouille, par une électrolyse parfaitement dirigée afin de donner 
à la couche d'étain une grande solidité en même temps qu'une uniformilté dans son épaisseur ont échoué jusqu'à présent.

Comme nouveau matériel il faut penser à l'aluminium que l'on peut fabriquer aujourd'hui très pur pour avoir une résistance suffisante, et qui aura, ainsi, les meilleures chances de se substituer au fer étamé.

En ce qui concerne la forme pratique et la grandeur des pots, le pot carré de 50 litres utilisé en Suède, garni d'une fermeture avec une pièce métallique en are pour assurer l'ocelusion parfaite (Patentbuegelverschluss) sera la meilleure, au point de vue idéal, sous tous les rapports.

$\Pi$ faut cependant tenir compte du particularisme des agriculteurs qui, dans tous les pays, sont très conservateurs.

Si nous nous tournons maintenant vers le deuxième temps du trans» port : celui qui amène le lait du dépôt central de ramassage à la laiterie de la Ville, nous allons trouver des conditions meilleures pour rendre ce transport plus éeonomique.

Que l'on se serve de l'automobile ou du chemin de fer comme moyen de transport, on peut dire, pour l'un comme pour l'autre, que le pot à lait n'est plus tout à fait de son temps. C'est une loi économique très connue que plus un emballage est grand, moins il est cher pour chaque unité de marchandise qu'il contient, ce que nous traduirons en disant qu'un pot à lait de 50 litres est, pour chaque litre de lait, moins cher qu'un pot de 20 litres. Les frais de transport, en même temps, sont moins chers proportionnellement pour les grands emballages que pour les petits.

Mais dans le transport du lait, le grand emballage joue encore un rôle plus important. Avec lui les pertes sont réduites fortement, le danger de l'infection par des contacts multipliés est diminué; les frais de nettoyage sont plus petits et la qualité du lait, surtout s'il fait chaud, sera mieux garantie, parce qu'une grande quantité s'échauffe beaucoup plus lentement qu'une petite.

Dans les centres de ramassage, on réunit, puisque c'est là le but de ces dépôts, de grandes quantités de lait; il y est donc très possible de se servir de grands emballages, et on aura raison de le faire.

La question se pose maintenant de savoir quelle sera la forme de cet emballage. Trois sortes ont déjà fait l'objet d'expériences:

$1^{0}$ Le tonneau à lait de 100 à 150 litres;

$2^{\circ}$ L'automobile-citerne (tank) de 3.000 à 5.000 litres;

$3^{\text {o }}$ Le wagon-eiterne de chemin de fer de 5.000 à 10.000 litres.

Nous pouvons encore signaler qu'on expérimente actuellement le wagon-citerne de chemin de fer d'une capacité moindre, de 1.000 à 1.500 litres.

A ma connaissance, le tonneau à lait (fig I) s'est développé d'abord en Allemagne où une grande partie des laiteries le regarde comme un 
progrès. Ce tonneau est copié sur le tonneau de benzine en fer, mais il a un couvercle latéral qu'on peut enlever, il est garni d'une bague en caoutchouc et d'une bague de tension pour assurer la fermeture. Au début on s'est servi du fer étamé comme matériel de construction, puis de fer avec un revêtement intérieur en aluminium, et enfin, dans ces derniers temps, d'aluminium pur. On projette une nouvelle construction qui serait le tonneau de fer avec un revêtement d'aluminium et une garniture en amiante pour l'isolation.

L'usage de ce tonneau suppose, en général, que les dépôts centraux ont un quai, mais on peut très bien aussi se servir d'un appareil de levage, tels que ceux qui sont employés dans beaucoupd'autres branches del'industrie. Les tonneaux sont soulevés par des petites grues. Dans ces derniers temps; on peut effectuer leur vidange, soit avec une pompe aspirante, soit au moyen de l'air comprimé.

On choisira partout le tonneau de 100 à 150 litres, toutes les fois qu'il s'agira de quantités de lait pas trop grandes, ou bien lorsque des difficultés contrarieront l'emploi des wagonsciternes, alors même que

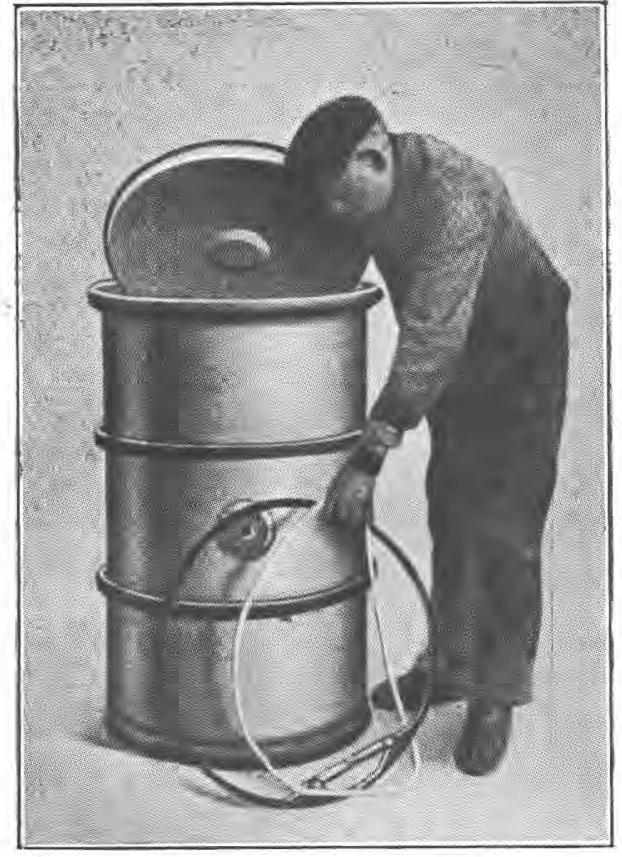

Fig. 1. - Tonnead a LatT. dans ce cas on aurait en face de soi de grandes quantités de lait.

Le wagon-citerne doit son développement rapide à l'augmentation également rapide de la consommation du lait dans les grandes villes des Etats-Unis. Il fut précédé d'ailleurs de l'automobile-citerne.

La fig. 2 montre une citerne en acier, émaillée à l'intérieur et garnie en dehors d'un revêtement en liège de 2 pouces, recouvert lui-même d'une enveloppe de sûreté en tôle. Pour charger et pour vider la citerne, on se sert de deux tubulures et pour la nettoyer, elle est garnie d'un trou d'homme. La vidange en est presque toujours effectuée par l'air comprimé qui arrive d'abord par en bas, afin de bien mélanger le lait, puis il se collecte en haut et fait pression ensuite pour aider à l'évacuation du récipient.

Cette riterne est montée d'une manière fixe sur le châssis de l'auto- 
mobile. Vide, pour 4.000 litres, elle pèse environ $1.100 \mathrm{~kg}$. montée entièrement et chargée de lait à plein, elle pèse $7.000 \mathrm{~kg}$.

La maison ScotT Powell à Philadelphie (1) va chercher les 120.000 litres de lait dont elle a besoin journellement dans des points qui sont éloignés parfois de $120 \mathrm{~km}$. Elle utilise de pareilles automobilesciternes. Avec l'air comprimé à 3,85 atmosphères, elle monte $685 \mathrm{~kg}$. de lait par minute à une hauteur de $10 \mathrm{~m}$. Autrefois, si on avait eu recours à des pots, pour la même quantitéde lait, il aurait fallu 7 hommes pour la manipulation nécessitée, alors qu'aujourd'hui une seule personne suffit.

Les frais de déchargement de lait, pour 1.000 livres $(453 \mathrm{~kg}$., soit 440 litres) se montaient autrefois à 44 cents (11 francs au cours actuels) mais aujourd'hui, ils atteignent 1 cent.

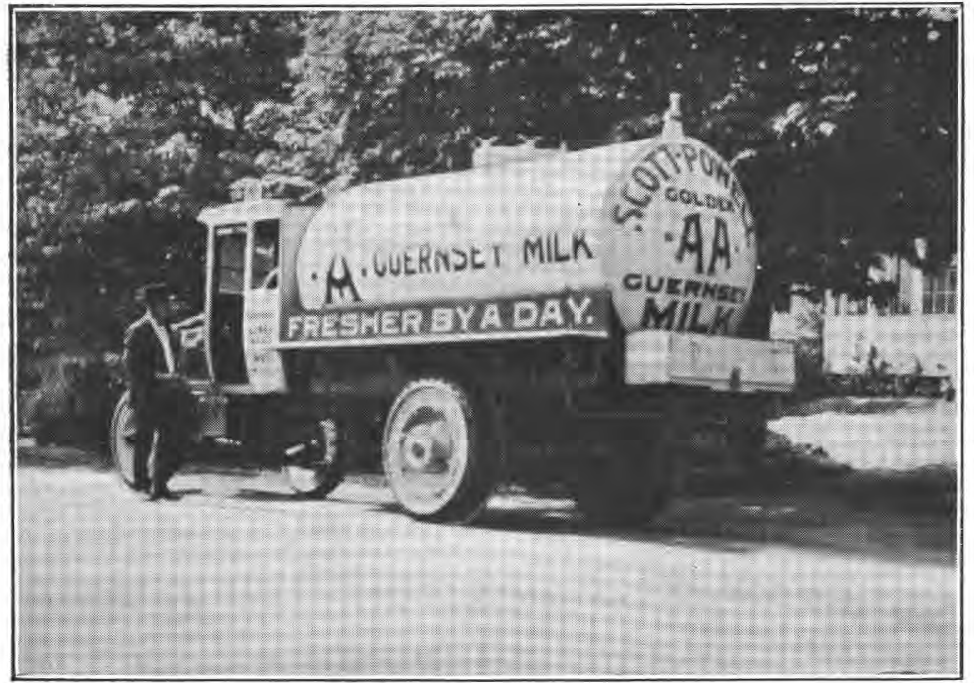

Fig. 2. - Automobile-Citerne.

Dans d'autres grandes villes américaines, des établissements laitiers ont augmenté leur parc d'automobiles-citernes et possèdent jusqu'à 40 voitures.

Economiquement parlant, l'emploi des automobiles-citernes permet d'augmenter le rayon d'action qui est ainsi plus grand si on le compare à celui qu'assurerait le chemin de fer. Ici, en effet, le déchargement dans les gares avec ses prix relativement élevés fait défaut. Nous ajouterons qu'il n'y a plus à se préoccuper des horaires des chemins de fer, parfois difficiles à suivre.

(1) D'après Lichtenberger ; "Die Milchwirtschaft der U. S. A. ” publié par a Molkerei-Zeitung n, Hildesh эim. 


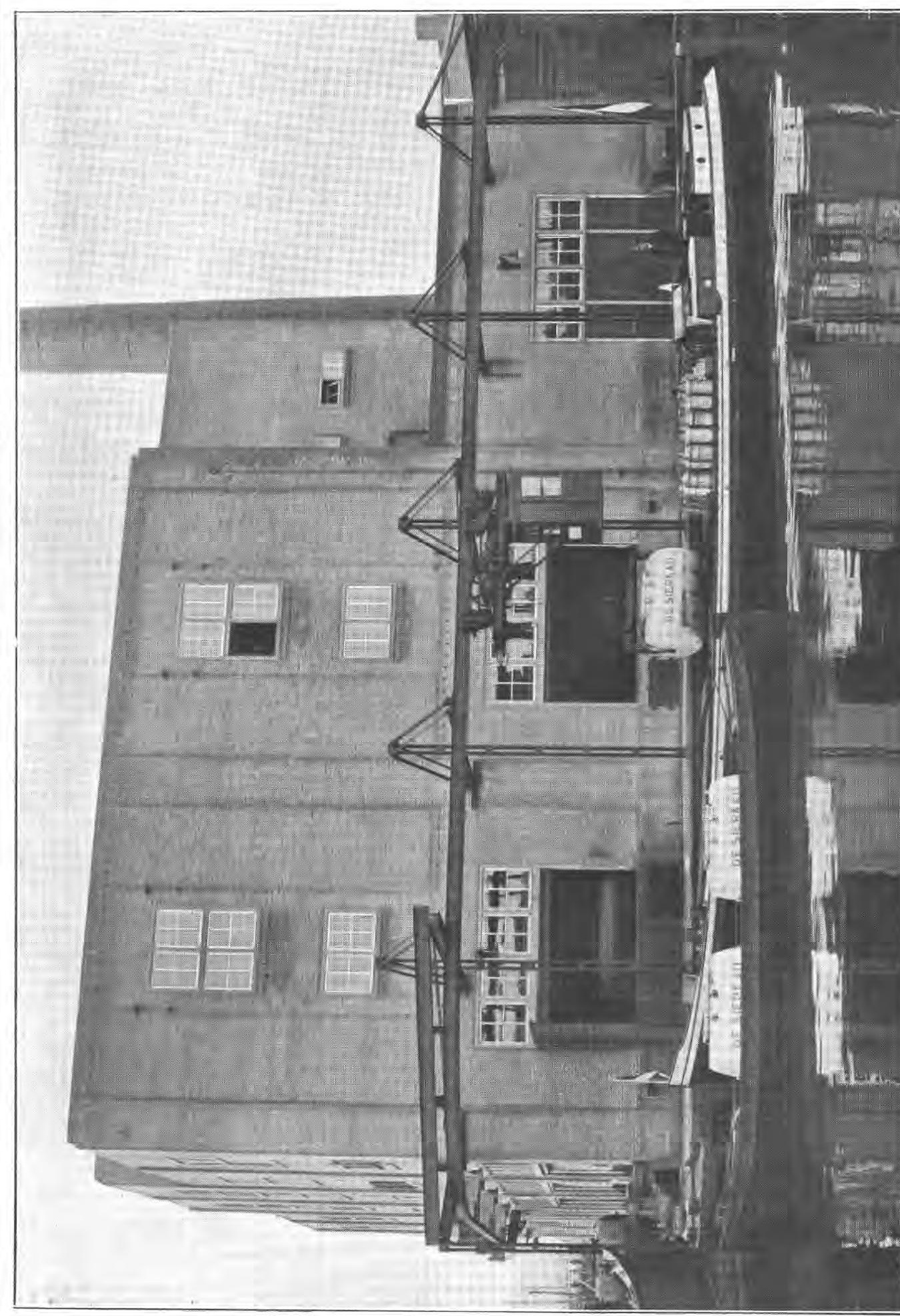


Enfin, le lait arrive plus vite à la laiterie et conséquemmerit, plus vite au consommateur.

Une laiterie de la Pensylvanie, estime qu'en substituant le transport du lait par automobile-citernes, au transport, par pots, elle a réalisé une économie de 20 dollars au (moins $500 \mathrm{fr}$. au cours actuel) par automobile et par jour.

En Allemagne, il y a pour l'instant en service deux automobilesciternes qui sont faites en aluminium.

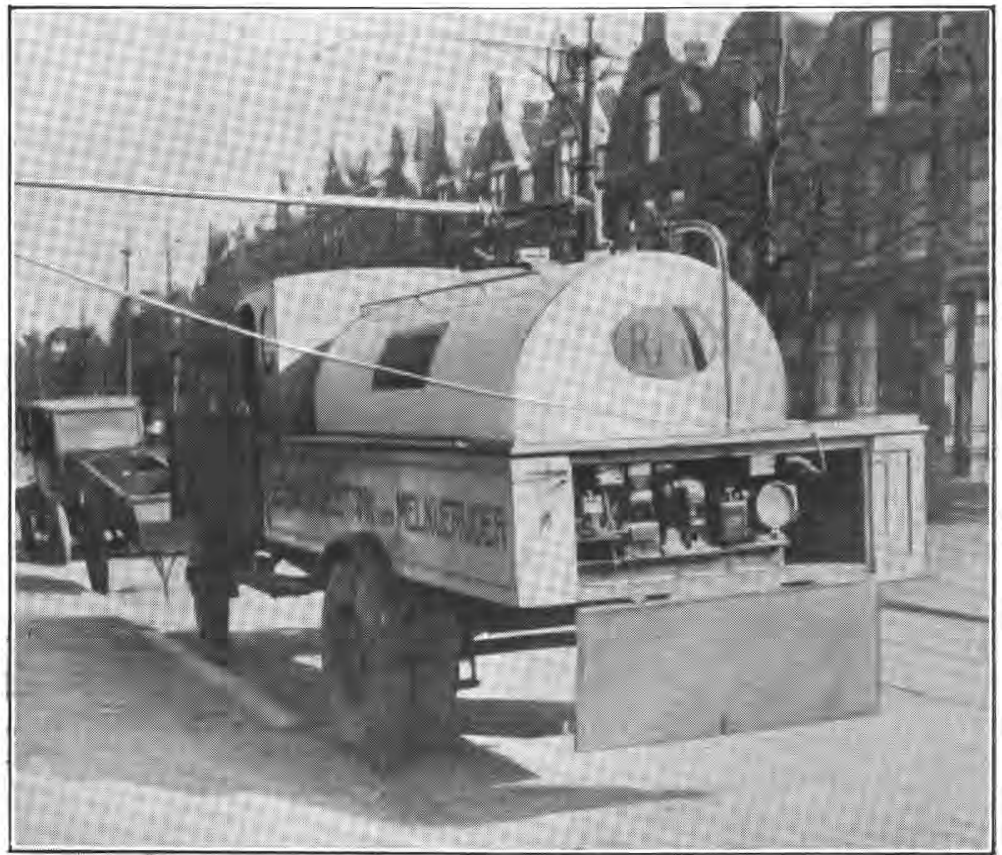

Fig. 4. - Automobile-Citerne aveo vidange a l'atr comprimé.

Aux Pays-Bas, la laiterie de Sierkan, à La Haye, utilise des citernes mises sur bateau pour aller chercher son lait. Comme la fig. 3 le montre, lorsque le lait est arrivé devant l'entrée, les citernes sont levées à l'aide d'une grue et portées à la laiterie pour la vidange. Les mêmes citernes servent également pour distribuer le lait pasteurisé et refroidi entre les nombreuses succursales de la Ville où l'on remplit les pots pour la vente.

Les citernes de cette firme ne sont pas isolées; elles sont en fer étamé et peuvent être chargées de 2.000 litres de lait.

Nous citerons une autre laiterie de Rotterdam, qui utilise des automobiles-citernes en acier émaillé d'un modèle américair, mais de fabrication hollandaise pour la distribution du lait. 
Ainsi que le montre la fig. 4, ces automobiles sont munies d'un petit compresseur à air, sans lubrification, grâce auquel le lait est poussée à travers une conduite jusqu'à la succursale.

Envisageons maintenant le transport de grandes quantités de lait:

Nous pouvons avoir à notre disposition le wagon-citerne des compagnies de chemins de fer; mais présentement il n'est guère utilisé qu'aux Etats-Unis, où de tels wagons portent deux citernes de 12.000 litres.

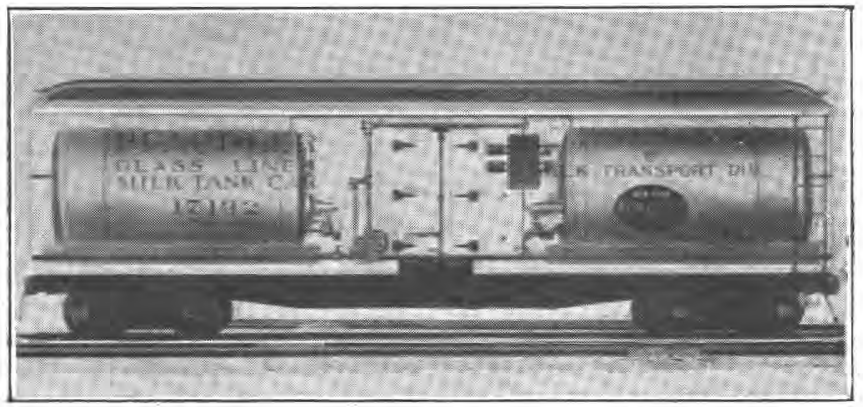

Fig. 5. - Vagon-Citerne de Chemin de fer.

Ces citernes sont munies d'un agitateur pour rendre le lait homogène, d'un dispositif d'éclairage, de thermomètres et d'une pompe à lait à impulsion électrique. Le courant est pris à la station de décharge à l'aide d'un câble.

Un désavantage de ces citernes est la nécessité de devoir les remplir entièrement. Le lait souffre d'une vidange partielle, aussi dans les wagonsciternes, a-t-on laissé un peu de place à côté des citernes, afin qu'on puisse y mettre des pots qui recueillent le lait en excédent après le remplissage complet des citernes.

Voici quelques chiffres donnant une idée des économies réalisées par l'emploi des wagons-citernes :

La "Harmony Creamery " à Pittsburg estimait ses pertes de lait, pendant 151 jours d'hiver à 45 gallons (plus de 200 litres) par jour quand le transport se faisait dans les pots, et pendant les 294 jours de l'année restants, les pertes atteignaient 80 gallons, soit plus de 400 litres par jour. Ces pertes, à la fin de l'année se montaient à environ 4.000 dollars; elles s'atténuèrent lorsque, à partir de 1922, la laiterie utilisa les wagonsciternes des chemins de fer. Mais ce n'est pas tout, car sur la réfrigération par la glace, on fit des économies de 4.000 dollars (100.000 fr.), sur la main-d'œuvre ouvrière de 15.000 dollars et sur les pertes en ait de 4.300 dollars, au total près de 600.000 francs.

On fit encore des économies sur l'emploi des automobiles et sur le nettoyage. Bref, pour un transport annuel de 1.389.260 gallons de lait, 
soit plus de 2.250 .000 de litres, pour 3 wagons-citernes d'une contenance de 20.000 litres, la laiterie a enregistré une économie totale atteignant 35.265.94 dollars, soit en franes, à 25,20 le dollar au cours actuel, près de 900.000 francs.

A travers tous ces chiffres, on n'a pas encore pris en considération que la durée du service était diminuée de $1 \mathrm{~h} .1 / 2$ que la capacité de la laiterie a pu être augmentée de 227.910 gallons, soit 1.026 .000 litres environ, sans qu'il y ait plus d'encombrement; que la qualité du lait était améliorée de $30 \%$ environ et qu'on faisait aussi des économies sur la vapeur, sur l'électricité, sur les appareils en service.

Actuellement, près de 100 wagons-citernes sont utilisés aux EtatsUnis; ils appartiennent, soit à des laiteries, soit à de grandes sociétés de transport qui louent leurs wagons-citernes.

Mais il n'est pas toujours possible d'utiliser des citernes aussi grandes. C'est pour cela qu'on a pensé à employer des petites citernes de 1.000 à 2.000 litres pour le transport par chemin de fer. Les études réclamées par ce problème n'ont pas encore dépassé la phase d'essai et les recherches que j'ai fait faire en Allemagne sur ce point, n'ont pas encore eu un résultat satisfaisant,

Les petites citernes seraient, dans ce cas, placées dans une caisse et on ne mettrait 6 ou 8 sur un wagon à plateforme découverte, l'une à côté de l'autre.

La construction des petites citernes a permis de montrer que celles-ci étaient d'un bon service, mais toutes les difficultés de leur emploi ne sont pas encore résolues, et il reste celle d'élever rapidement les caisses qui les renferment, et qui sont assez lourdes, de l'automobile pour les placer sur le wagon, dans les petites gares. A mon avis, cette difficulté pourrait être évitée par un bon équipement de ces dernières.

Le dernier temps du transport du lait est enfin celui dans lequel cet aliment va de la laiterie à la boutique de vente et au ménage du consommateur.

Cette vente est souvent libre et nous estimons qu'elle est à désavouer, surtout si elle est faite dans la rue.

D'après moi, dans l'avenir il n'y a qu'une seule méthode de transport pour ce troisième temps, c'est celle du flacon à lait.

Les Etats-Unis ont fait de très grands progrès, et avec succès, dans cette direction. Nul doute que les autres nations ne les suivent dans l'intérêt de l'hygiène.

Certainement, la vente en bouteilles est plus chère que la vente libre, dans le premier récipient venu, mais les consommateurs doivent savoir qu'il est de leur intérêt de recourir à une pareille amélioration.

Une des conditions essentielles pour faciliter le transport du lait en bouteilles, est que les flacons aient tous la même dimension. La standardisation des bouteilles à lait est un fait accompli aux Etats-Unis 
et elle sera réalisée en Allemagne cette année. Le flacon normal, dans des caisses normales: voilà ce qui facilite également le travail dans la laiterie. L'exploitation dans tous les services est améliorée, ainsi que le mode de distribution et connaissant l'encombrement des caisses, il devient possible d'organiser comme il faut leur convoiement dans la laiterie.

Les voitures qui font la livraison directe au consommateur doivent être construites de façon qu'on puisse y mettre 400 litres de lait en bouteilles, tandis que les voitures qui font la livraison en gros et qui sont bien isolées doivent pouvoir contenir de 1.000 à 3.000 litres.

La distribution du lait en bouteilles nous donne également la possibilité de diminuer les frais des succursales dans les grandes villes. On peut en effet, entrevoir la possibilité de diminuer le nombre de chambres frigorifiques et, avec elles, le travail de déchargement qui s'y trouve intimement lié. A notre avis, le lait en bouteilles serait laissé le soir dans les grandes automobiles isolées logées dans les cours des succursales pour être distribué le lendemain directement aux voitures de détail.

Tous les progrès réalisés dans ces dernières années dans le transport du lait, à quelque temps que ce soit, sont déjà grands et, à n'en pas douter, il faciliteront, par leur développement, l'exploitation de la laiterie dans les grandes villes, en même temps qu'ils permettront aux consommateurs de recevoir un lait à boire encore meilleur.

\title{
CONTRIBUTION A L'ÉTUDE DU LAIT STÉRILISÉ
}

\author{
par M. PELLET, \\ Ingénieur agronome.
}

La stéritisation du lait a été jusqu'ici pratiquée avec un empirisme incompatible avec la constance des résultats. Les méthodes très variées s'inspirent davantage de préférences personnelles que de règles scientifiquement établies. La stérilisation absolue ne peut être obtenue sans altérer les qual tés physiques et biologiques du lait; elle ne doit avoir d'autre objectif que la sécurité et la conservation dans des limites normales d'emploi. Les autoclaves sont d'un maniement plus délicat qu'on ne se le figure. Echauffement de l'atmosphère intérieure, relations avec la pression, distribution de la vapeur, température au sein des liquides traités, durée effective de la stérilisation, sont autant de points dont on s'est peu inquiété. Sollicité par l'étude de ces divers états du problème, j'avais exposé au Congrès International de Laiterie (Mai 1926) une technique issue de mes observations à la Laiterie municipale de Nantes.

Simultanément, M. Pellet donna t dans le "Lait" (Septembre 1926, $\left.N^{\circ} 58\right)$ un important travail sur la Marche de la température dans un stérilisateur industriel. 